\title{
A Method of Upgrading a Hydrostatic Model to a Nonhydrostatic Model
}

\author{
Chi-Sann Liou *, Yang-Fan Sheng, Tzay-Ming Leou, Shwu-Ching Lin, Tien-Chiang Yeh, \\ Chuen-Teyr Terng, Der-Song Chen, Kang-Ning Huang, and Mei-Yu Chang \\ Central Weather Bureau, Taipei, Taiwan, ROC
}

Received 11 March 2008, accepted 24 September 2008

\begin{abstract}
As the sigma-p coordinate under hydrostatic approximation can be interpreted as the mass coordinate without the hydrostatic approximation, we propose a method that upgrades a hydrostatic model to a nonhydrostatic model with relatively less effort. The method adds to the primitive equations the extra terms omitted by the hydrostatic approximation and two prognostic equations for vertical speed $w$ and nonhydrostatic part pressure $p^{\prime}$. With properly formulated governing equations, at each time step, the dynamic part of the model is first integrated as that for the original hydrostatic model and then nonhydrostatic contributions are added as corrections to the hydrostatic solutions. In applying physical parameterizations after the dynamic part integration, all physics packages of the original hydrostatic model can be directly used in the nonhydrostatic model, since the upgraded nonhydrostatic model shares the same vertical coordinates with the original hydrostatic model. In this way, the majority codes of the nonhydrostatic model come from the original hydrostatic model. The extra codes are only needed for the calculation additional to the primitive equations. In order to handle sound waves, we use smaller time steps in the nonhydrostatic part dynamic time integration with a split-explicit scheme for horizontal momentum and temperature and a semi-implicit scheme for $w$ and $p^{\prime}$. Simulations of 2-dimensional mountain waves and density flows associated with a cold bubble have been used to test the method. The idealized case tests demonstrate that the proposed method realistically simulates the nonhydrostatic effects on different atmospheric circulations that are revealed in theoretical solutions and simulations from other nonhydrostatic models. This method can be used in upgrading any global or mesoscale models from a hydrostatic to nonhydrostatic model.
\end{abstract}

Key words: Nonhydrostatic model, Hydrostatic to nonhydrostatic

Citation: Liou, C. S., Y. F. Sheng, T. M. Leou, S. C. Lin, T. C. Yeh, C. T. Terng, D. S. Chen, K. N. Huang, and M. Y. Chang, 2009: A method of upgrading a hydrostatic model to a nonhydrostatic model. Terr. Atmos. Ocean. Sci., 20, 727-739, doi: 10.3319/TAO.2008.09.24.01(A)

\section{INTRODUCTION}

The primitive equations used as governing equations in atmospheric hydrostatic models include hydrostatic approximation that replaces the vertical momentum equation by the hydrostatic balance equation. The approximation is very accurate when vertical velocity, and hence vertical acceleration, is very small. The hydrostatic approximation not only simplifies the governing equations but also eliminates sound waves from the solution to avoid the necessity in handling those fast moving waves in time integration. However, if one needs to simulate atmospheric circulation with strong vertical motions, such as thunderstorms or hurricanes, a nonhydrostatic model may be desired. In this paper,

\footnotetext{
* Corresponding author

E-mail:lioucs@yahoo.com
}

we describe a method that extends a hydrostatic model to a nonhydrostatic model by adding the terms and equations neglected by the hydrostatic approximation to the primitive equations as corrections to the dynamic part solution at each time step. With this approach, the model can be run as a hydrostatic or nonhydrostatic model depending upon the user's choice for applications. The only approximation made in this method is to linearize the pressure tendency equation to formulate a stable scheme for the nonhydrostatic part time integration. Since sound wave prediction may be distorted by linearization, nonhydrostatic models developed by this method are not suitable to simulate sound wave dominated phenomena. However, they should be perfectly adequate for numerical weather prediction (NWP) since sound waves play an insignificant role on weather changes. 
For a given temperature profile, the hydrostatic approximation makes height and pressure monotonically depend on each other so that pressure can be used as a vertical coordinate. To better handle bottom boundary conditions, the terrain-following sigma-p coordinate, defined as $\sigma=\left(p-p_{T o p}\right) /$ $\left(p_{\text {Surface }}-p_{\text {Top }}\right)$, is usually used as the vertical coordinate in hydrostatic models. Laprise (1992) introduced a new concept that if we interpret the hydrostatically balanced part of pressure as the air mass weight above the area, the sigma-p coordinate can still be used in nonhydrostatic models and it is called the mass coordinate. When interpreting the hydrostatic pressure as air mass weight per area, we can formulate the governing equations for a fully compressible atmosphere without the hydrostatic approximation in the mass coordinates. Consequently, the governing equations, and hence the whole codes, of a hydrostatic model can be used as the major part of a nonhydrostatic model extended from the hydrostatic model. In the dynamic integration of the nonhydrostatic model, after the hydrostatic tendency has been calculated the nonhydrostatic tendency from terms and equations omitted by the hydrostatic approximation can then be added to complete the time step. This correction type time integration for the dynamic part works as the time splitting integration, with which different terms are integrated by different numerical methods. In the physics part time integration after the dynamic integration at each time step, all physical parameterization packages of the original hydrostatic model can be directly applied for the nonhydrostatic model as they share the same vertical coordinate. The challenge in this approach is to organize the governing equations into a form that hydrostatic and nonhydrostatic terms are separable, and to select proper prognostic variables and numerical schemes for which the time integration is stable and efficient. The Weather Research Forecasting (WRF) model also uses mass coordinates in its two dynamic cores: NMM developed at the National Centers for Environmental Prediction (NCEP, Janjic et al. 2001) and ARW developed at the National Center for Atmospheric Research (NCAR, Klemp et al. 2007). However, we choose different prognostic variables and different numerical methods in handling sound waves. These differences will be discussed in section 4 .

There are other vertical coordinates used in nonhydrostatic models, such as the sigma-z coordinate in the Coupled Ocean/Atmosphere Mesoscale Prediction System (COAMPS, Hodur 1997) and the sigma-p* coordinate in the FifthGeneration Penn State/NCAR Mesoscale Model (MM5, Dudhia 1993) and the Purdue Mesoscale Model (PMM, Hsu and Sun 2001). The sigma-p* coordinate defines the vertical coordinate by a reference surface pressure $\mathrm{p}^{*}$ that is constant in time and varies in height only. Therefore, the sigma-p* coordinate is equivalent to the sigma- $z$ coordinate. The sigma- $z$ coordinate has an advantage in that the coordinate is not changed with time. However, the sigma-z coordinate involves with a rigid upper lid that can artificially increase pressure or reduce mass (Klemp et al. 2007). In the MM5, the non-conserving problem is minimized by ignoring diabatic terms in the pressure prediction equation (Dudhia 1993). The mass coordinate is favored over the sigma- $\mathrm{z}$ coordinate in the WRF-ARW dynamic core because of mass conservation and convenience in switching to hydrostatic integration (Klemp et al. 2007).

\section{GOVERNING EQUATIONS}

For a hydrostatic model, the primitive equations in the sigma-p coordinates can be written as:

$$
\begin{aligned}
& \frac{d \vec{V}}{d t}+f \vec{k} \times \vec{V}+R T \nabla \ln \pi_{s}+\nabla \phi=\vec{F}_{r} \\
& \frac{d T}{d t}-\frac{R T}{C_{p}} \frac{d \ln \pi_{s}}{d t}=\frac{Q_{T}}{C_{p}} \\
& \frac{d \pi_{s}}{d t}+\pi_{s} \nabla \cdot \vec{V}+\pi_{s} \frac{\partial \dot{\sigma}}{\partial \sigma}=0 \\
& \frac{d q}{d t}=S \\
& \frac{\partial \phi}{\partial \ln \sigma}=-R T
\end{aligned}
$$

where $\frac{d}{d t}=\frac{\partial}{\partial t}+\vec{V} \cdot \nabla+\dot{\sigma} \frac{\partial}{\partial \sigma}, \pi_{s}=p_{\text {surface }}, \sigma=p / \pi_{s}, \vec{V}$ is horizontal wind velocity, $f$ is the Coriolis parameter, $f=$ $2 \Omega \sin \varphi, \phi$ is the geopotential of a sigma-p level, $\phi=g z_{s}-$ $\int_{1}^{\sigma} R T d \ln \sigma, Q_{T}$ is diabatic heating rate, $\vec{F}_{r}$ is momentum friction, and $S$ is moisture source. For simplicity we have chosen $p_{T o p}=0$ in defining the sigma-p coordinate. Equation (3) is a form of the continuity equation derived from the transformed continuity equation for any general coordinate $\eta$ (Kasahara 1974):

$\frac{d}{d t}\left(\ln \frac{\partial p}{\partial \eta}\right)+\nabla_{\eta} \cdot \vec{V}+\frac{\partial \dot{\eta}}{\partial \eta}=0$

The hydrostatic balance Eq. (5) is the vertical momentum equation with the hydrostatic approximation, i.e., $d w / d t \approx$ 0 . The pressure tendency equation becomes a trivial equation with the hydrostatic approximation (see Appendix) and the pressure can be diagnosed from the surface pressure $\pi_{s}$ as $p=\pi_{s} \sigma$.

To add nonhydrostatic components to the primitive 
equations, we select vertical velocity $(w)$ and the nonhydrostatic part of pressure $\left(p^{\prime}\right)$, defined as the difference between total pressure and hydrostatic pressure, as two extra prognostic variables. Following Laprise (1992), we can write the governing equations without the hydrostatic approximation in the mass coordinates as:

$\frac{d \vec{V}}{d t}+f \vec{k} \times \vec{V}+R T \nabla \ln p+\frac{1}{\pi_{s}} \frac{\partial p}{\partial \sigma} \nabla \Phi+\tilde{f} w \vec{I}_{E}=\vec{F}_{r}$

$\frac{d T}{d t}-\frac{R T}{C_{p}} \frac{d \ln p}{d t}=\frac{Q_{T}}{C_{p}}$

$\frac{d \pi_{s}}{d t}+\pi_{s} \nabla \cdot \vec{V}+\pi_{s} \frac{\partial \dot{\sigma}}{\partial \sigma}=0$

$\frac{d w}{d t}+g\left(1-\frac{1}{\pi_{s}} \frac{\partial p}{\partial \sigma}\right)-\tilde{f} \vec{V} \cdot \vec{I}_{E}=F_{w}$

$\frac{d \ln p}{d t}+\frac{C_{p}}{C_{v}} D_{3}=\frac{Q_{T}}{C_{v} T}$

$\frac{d q}{d t}=S$

where $w$ is vertical speed, $\pi$ is hydrostatic pressure, $\pi=$ $\int_{z}^{\infty}(\rho g) d z, \sigma=\pi / \pi_{s}, p$ is total pressure, $p=\pi+p^{\prime}, \Phi=g z_{s}-$ $\pi_{s} \int_{1}^{\sigma} \frac{d \sigma}{\rho}, \tilde{f}=2 \Omega \cos \varphi, \vec{I}_{E}$ is a unit vector defined as the cross product of the unit vector in y-direction and unit vector in north-direction, and $D_{3}$ is three dimensional divergence, $D_{3} \equiv \nabla_{3} \cdot \vec{V}_{3}=\nabla \cdot \vec{V}+\frac{\rho}{\pi_{s}}(\nabla \phi) \cdot \frac{\partial \vec{V}}{\partial \sigma}-\frac{\rho g}{\pi_{s}} \frac{\partial w}{\partial \sigma}$.

The pressure tendency Eq. (10) involves the very delicate balance between heating and divergence. We can rewrite this equation in terms of hydrostatic and nonhydrostatic pressure as:

$\frac{d\left(\pi+p^{\prime}\right)}{d t}+\left(\pi+p^{\prime}\right) \frac{C_{p}}{C_{v}} D_{3}=\left(\pi+p^{\prime}\right) \frac{Q_{T}}{C_{v} T}$

Since $\pi$ is much greater than $p^{\prime}$ for circulation related to weather prediction and $d p^{\prime} / d t$ is important mainly for sound waves, we simplify this equation by first assuming the nonhydrostatic part of pressure plays no significant role on the hydrostatic part of pressure (or mass) tendency calculation. As demonstrated in the appendix, in the mass coordinates, if only the hydrostatic part of pressure is used in cal- culating $D_{3}$ by Eq. (A7), a trivial equation for the hydrostatic pressure tendency can be obtained by adding the continuity and thermodynamic equations together as:

$\frac{d \pi}{d t}+\pi \frac{C_{p}}{C_{v}} D_{3}=\pi \frac{Q_{T}}{C_{v} T}$

By subtracting Eq. (13) from Eq. (12), a nonhydrostatic pressure tendency equation can be obtained as:

$\frac{d p^{\prime}}{d t}+p^{\prime} \frac{C_{p}}{C_{v}} D_{3}=p^{\prime} \frac{Q_{T}}{C_{v} T}$

Since Eq. (13) is a trivial equation, it will not be included in the time integration and the pressure tendency Eq. (12) is simplified to Eq. (14) under this assumption. We further linearize Eq. (14) by replacing $p^{\prime}$ in the second term with a time-independent reference pressure $\tilde{p}$ as:

$\frac{d p^{\prime}}{d t}+\tilde{p} \frac{C_{p}}{C_{v}} D_{3} \approx p^{\prime} \frac{Q_{T}}{C_{v} T}$

The first simplification is equivalent to assuming the hydrostatic and nonhydrostatic pressure tendencies are balanced independently. The further linearization made in Eq. (15) is to formulate a stable semi-implicit scheme for integrating $d w / d t$ and $d p^{\prime} / d t$ equations. By substituting the pressure-tendency Eqs. (13) and (15) into the thermodynamic equation $\frac{d T}{d t}+\frac{R T}{C_{v}} D_{3}=\frac{Q_{T}}{C_{v}}$, we find the temperature tendency equation to be:

$\frac{d T}{d t}-\frac{R T}{C_{p}} \frac{d \ln p}{d t}=\frac{Q_{T}}{C_{p}}+\frac{\left(\tilde{p}-p^{\prime}\right)}{p} \frac{R T D_{3}}{C_{v}}$

Therefore, we need to add $\frac{\left(\tilde{p}-p^{\prime}\right)}{p} \frac{R T D_{3}}{C_{v}}$ to the temperature tendency equation for energy conservation after linearizing the nonhydrostatic pressure tendency equation.

With the simplification, the extra terms and equations added to the primitive equations for a nonhydrostatic model can be summarized as:

$$
\begin{aligned}
& \frac{\partial \vec{V}}{\partial t}+\frac{R T}{p}\left(-p^{\prime} \nabla \ln \pi_{s}+\nabla p^{\prime}\right)+\frac{1}{\pi_{s}} \frac{\partial p^{\prime}}{\partial \sigma} \nabla \Phi \\
& +\nabla \phi^{\prime}+\tilde{f} w \vec{I}_{E}=\left(\frac{d \vec{V}}{d t}\right)_{H}+\vec{F}_{r}
\end{aligned}
$$


$\frac{\partial T}{\partial t}-\frac{R T}{C_{p} p}\left(-p^{\prime} \frac{d \ln \pi_{s}}{d t}+\frac{d p^{\prime}}{d t}\right)=\frac{\left(\tilde{p}-p^{\prime}\right) R T D_{3}}{p C_{v}}$

$+\left(\frac{d T}{d t}\right)_{H}+\frac{Q_{T}}{C_{p}}$

$\frac{d w}{d t}+\frac{g}{\pi_{s}} \frac{\partial p^{\prime}}{\partial \sigma}-\tilde{f} \vec{V} \cdot \vec{I}_{E}=F_{w}$

$\frac{d p^{\prime}}{d t}+\tilde{p} \frac{C_{p}}{C_{v}} D_{3}=p^{\prime} \frac{Q_{T}}{C_{v} T}$

$\phi^{\prime}=\int_{1}^{\sigma}\left(\frac{R T p^{\prime}}{p}\right) d \ln \sigma$

where $\left(\frac{d \vec{V}}{d t}\right)_{H} \equiv-f \vec{k} \times \vec{V}-R T \nabla \ln \pi_{s}-\nabla \phi$ and $\left(\frac{d T}{d t}\right)_{H} \equiv$ $\frac{R T}{C_{p}} \frac{d \ln \pi_{s}}{d t}$ denote the hydrostatic tendencies contributed by the dry dynamics and $\phi^{\prime} \equiv \Phi-\phi$ denotes the correction to the geopotential due to nonhydrostatic effects. Equations (17) to (21) together with Eqs. (1) to (5) form a complete set of governing equations for a fully compressible atmosphere with the only approximation being to linearize the pressure tendency equation, which is important to sound waves only. We found that a choice of $\tilde{p}$ to be $10 \%$ of the standard atmosphere pressure gives very acceptable results both in idealized case simulations (see below) and real data forecasts.

\section{TIME INTEGRATION}

For the time integration of the nonhydrostatic model developed in this approach, the dry dynamic part of the hydrostatic Eqs. (1) to (4) is first integrated as they are done in the original hydrostatic model. In our original hydrostatic model, the governing Eqs. (1) to (4) are numerically solved by fourth order central differencing in the horizontal on staggered $\mathrm{C}$ grids and second order central differencing in the vertical on staggered sigma levels. The vertical staggering carries vertical velocity at the full levels and all other variables at the half levels. The time integration is conducted by the leapfrog scheme together with an explicit time-split scheme (Madala 1981) to handle fast moving gravity waves. The time-split scheme decomposes gravity wave related variables, horizontal divergence and pseudo geopotential, into vertical modes. It then uses the same leapfrog scheme but smaller time steps to compute corrections to the first few fast moving modes to maintain their stability. Rather than damping those fast moving gravity wave modes by an implicit scheme, as is normally done in a semi-implicit scheme, the Madala scheme includes those fast moving gravity waves in the model solution during time integration.

If model integration is selected to include nonhydrostatic components, dry dynamic hydrostatic tendencies $\left(\frac{d \vec{V}}{d t}\right)_{H}$ and $\left(\frac{d T}{d t}\right)_{H}$ are first calculated by the difference after and before the dynamic integration divided by the length of the time step. Equations (17) to (20) are then integrated with a suitable method to handle fast moving sound waves. In our development, we use an explicit-split scheme to integrate Eqs. (17), (18), and the horizontal part Eq. (20) and a semi-implicit scheme to integrate Eq. (19) and the vertical part Eq. (20). Since our original hydrostatic model is integrated by a flux form of Eqs. (1) to (4), we will now express Eqs. (17) and (18) in a flux form as well. We rewrite the Eqs. (17) to (20) as:

$$
\begin{aligned}
& \frac{\partial\left(\pi_{s} u\right)}{\partial t}+\frac{\pi_{s}}{\rho} \frac{\partial p^{\prime}}{\partial x}+\frac{\partial \Phi}{\partial x} \frac{\partial p^{\prime}}{\partial \sigma}=R H_{u}+\pi_{s} F_{r u} \\
& \frac{\partial\left(\pi_{s} v\right)}{\partial t}+\frac{\pi_{s}}{\rho} \frac{\partial p^{\prime}}{\partial y}+\frac{\partial \Phi}{\partial y} \frac{\partial p^{\prime}}{\partial \sigma}=R H_{v}+\pi_{s} F_{r v} \\
& \frac{\partial\left(\pi_{s} T\right)}{\partial t}-\frac{1}{C_{p}} \frac{\pi_{s}}{\rho} \frac{\partial p^{\prime}}{\partial t}=R H_{T}+\frac{\pi_{s} Q_{T}}{C_{p}} \\
& \frac{\partial w}{\partial t}-\frac{g}{\pi_{s}} \frac{\partial p^{\prime}}{\partial \sigma}=R H_{w}+F_{r w} \\
& \frac{\partial p^{\prime}}{\partial t}-\frac{C_{p} \rho g}{C_{v} \pi_{s}} \tilde{p} \frac{\partial w}{\partial \sigma}+\frac{C_{p}}{C_{v}} \tilde{p}\left[\frac{\partial u}{\partial x}+\frac{\partial v}{\partial y}\right. \\
& \left.+\frac{\rho}{\pi_{s}}\left(\frac{\partial \Phi}{\partial x} \frac{\partial u}{\partial \sigma}+\frac{\partial \Phi}{\partial y} \frac{\partial v}{\partial \sigma}\right)\right]=R H_{p}+\frac{p^{\prime} Q_{T}}{C_{v} T}
\end{aligned}
$$

where

$$
\begin{aligned}
& R H_{u}=\left(\frac{\partial \pi_{s} u}{\partial t}\right)_{H}-\tilde{f} \pi_{s} w \cos \varpi-\pi_{s} \frac{\partial \phi^{\prime}}{\partial x}+\frac{p^{\prime}}{\rho} \frac{\partial \pi_{s}}{\partial x} \\
& -\frac{\pi_{s}}{\rho} \frac{\partial p^{\prime}}{\partial x}, \\
& R H_{v}=\left(\frac{\partial \pi_{s} v}{\partial t}\right)_{H}+\tilde{f} \pi_{s} w \sin \varpi-\pi_{s} \frac{\partial \phi^{\prime}}{\partial y}+\frac{p^{\prime}}{\rho} \frac{\partial \pi_{s}}{\partial y} \\
& -\frac{\pi_{s}}{\rho} \frac{\partial p^{\prime}}{\partial y}, \\
& R H_{T}=\left(\frac{\partial \pi_{s} T}{\partial t}\right)_{H}+\frac{1}{C_{p} \rho}\left[-p^{\prime}\left(\frac{\partial \pi_{s}}{\partial t}+u \frac{\partial \pi_{s}}{\partial x}+v \frac{\partial \pi_{s}}{\partial y}\right)\right. \\
& \left.+\pi_{s}\left(\frac{\partial p^{\prime}}{\partial t}+u \frac{\partial p^{\prime}}{\partial x}+v \frac{\partial p^{\prime}}{\partial y}+\dot{\sigma} \frac{\partial p^{\prime}}{\partial \sigma}\right)+\frac{C_{p} \pi_{s}}{C_{v}}\left(\tilde{p}-p^{\prime}\right) D_{3}\right],
\end{aligned}
$$




$$
\begin{aligned}
& R H_{w}=-\left(u \frac{\partial w}{\partial x}+v \frac{\partial w}{\partial y}+\dot{\sigma} \frac{\partial w}{\partial \sigma}\right)+\tilde{f}(u \cos \varpi-v \sin \varpi), \\
& R H_{p}=-\left(u \frac{\partial p^{\prime}}{\partial x}+v \frac{\partial p^{\prime}}{\partial y}+\dot{\sigma} \frac{\partial p^{\prime}}{\partial \sigma}\right)
\end{aligned}
$$

and $\varpi$ is the angle between $\mathrm{Y}$ and north directions.

The right hand side terms of the above equations are integrated with the time scheme used in the hydrostatic integration, as they are involved with Rossby and gravity waves. However, the left hand side terms are integrated with the following methods to handle sound waves. A forwardbackward scheme with a smaller time step is used in integrating the second and third terms of Eqs. (22) and (23) and the third term of Eq. (26). In this time integration, the second and third terms of Eqs. (22) and (23) are integrated forward and then the third term of Eq. (26) is integrated backward after the winds have been updated. An implicit scheme with the same small time step of the forwardbackward scheme is used in integrating the second terms of Eqs. (25) and (26). The implicit scheme integrates these terms by solving a tri-diagonal matrix. Once $p^{\prime}$ has been updated to the next regular time step, Eq. (24) can be easily integrated with $d p^{\prime} / d t$ computed from the $p^{\prime}$ change in this time step. Two or three smaller time steps per regular time step are usually enough to handle the sound wave integration. The smaller time step integration for the left hand side terms is illustrated with the following equations:

$$
\begin{aligned}
& \frac{\partial \tilde{u}}{\partial t}+h_{u}\left(p^{\prime}\right)=H_{u}, \\
& \frac{\partial \tilde{v}}{\partial t}+h_{v}\left(p^{\prime}\right)=H_{v}, \\
& \frac{\partial w}{d t}+\alpha \frac{\partial p^{\prime}}{\partial \sigma}=I,
\end{aligned}
$$

and

$$
\frac{\partial p^{\prime}}{\partial t}+\beta \frac{\partial w}{\partial \sigma}+m(\tilde{u}, \tilde{v})=J
$$

where $\tilde{u}$ represents $\left(\pi_{s} u\right), \widetilde{v}$ represents $\left(\pi_{s} v\right), h_{u}$ and $h_{v}$ represent the second and third terms of Eqs. (22) and (23), respectively, $H_{u}$ and $H_{v}$ represent the right hand side terms of Eqs. (22) and (23), $\alpha$ and $\beta$ represent the coefficients of $\partial / \partial \sigma$ derivatives in the second terms of Eqs. (25) and (26), $I$ and $J$ represent the right hand side terms of Eqs. (25) and (26), and $m$ represents the third term of Eq. (26). For a given regular time step $N$, the $(n+1)$ small time step integration at level $k$ can be written as:

$$
\begin{aligned}
& \tilde{u}_{k}^{n+1}=\tilde{u}_{k}^{n}+\delta t\left[-h_{u}\left(p_{k}^{\prime n}\right)+H_{u k}^{N}\right] \\
& \tilde{v}_{k}^{n+1}=\tilde{v}_{k}^{n}+\delta t\left[-h_{v}\left(p_{k}^{\prime n}\right)+H_{v k}^{N}\right] \\
& w_{k}^{n+1}=w_{k}^{n}+\delta t\left[\tilde{\alpha}_{k-1}^{N}\left(p_{k-1}^{\prime n^{*}}\right)-\tilde{\alpha}_{k}^{N}\left(p_{k}^{\prime n^{*}}\right)+I_{k}^{N}\right]
\end{aligned}
$$

and

$p_{k}^{\prime n+1}=p_{N}^{\prime n}+\delta t\left[\tilde{\beta}_{k}^{N}\left(w_{k}^{n^{*}}\right)-\tilde{\beta}_{k+1}^{N}\left(w_{k+1}^{n^{*}}\right)\right.$

$\left.-m\left(\tilde{u}_{k}^{n+1}, \tilde{v}_{k}^{n+1}\right)+J_{k}^{N}\right]$

where $\tilde{\alpha}$ and $\tilde{\beta}$ are $\alpha$ and $\beta$ weighted by layer thickness factors associated with the vertical differencing, and $p^{\prime^{*}}$ and $w^{n^{*}}$ are weighted averages between small time steps $n$ and $(n+1)$, i.e., $p^{\prime n^{*}}=v p^{\prime n+1}+(1-v) p^{\prime n}$ with $0.5 \leq v \leq 1$ representing the degree of implicitness. A default value of $v=0.8$ is typically used in the integration. As with all variables known at the time steps $N$ and $n, \tilde{u}^{n+1}$ and $\tilde{v}^{n+1}$ can be easily calculated by Eqs. (27) and (28). Once $\tilde{u}^{n+1}$ and $\tilde{v}^{n+1}$ are computed, the $m$ term of Eq. (30) can be evaluated and $w^{n+1}$ and $p^{\prime n+1}$ can be calculated by a tri-diagonal matrix solver after one or the other of them is eliminated through substitution. We choose to eliminate $w$ for solving $p^{\prime n+1}$ with boundary conditions $p^{\prime n+1}=0$ at the model top and $w=\vec{V} \cdot \nabla z_{s}$ at the surface. The linearization made in Eq. (15) or (20) allows us to formulate Eq. (30) without $p^{\prime}$ involved in the coefficient $\tilde{\beta}$.

\section{COMPARISON WITH THE WRF MODEL}

Both dynamic cores of the WRF model use the mass coordinates but with different prognostic variables and numerical methods. Similar to our choice, the NMM core chooses temperature, rather than potential temperature, as a prognostic variable for convenience in computing the gas law and applying physics packages. On the other hand, the ARW core chooses the potential temperature for convenience in energy conservation. However, the major differences among the two WRF dynamic cores and our method are in adding the nonhydrostatic contributions to the primitive equations. We add $w$ and $p^{\prime}$ as two additional prognostic variables, and add the third momentum Eq. (19) and nonhydrostatic pressure tendency Eq. (20) as two additional equations to close the system. The WRF-NMM core adds $\mathrm{w}$ and total pressure $\mathrm{p}$ as two additional prognostic variables. However, it adds three prognostic equations: the third momentum equation, pressure tendency equation, and the definition of $w g=\frac{d \Phi}{d t}$ together with the constraint of $\Phi=\Phi_{\mathrm{s}}+\pi_{s} \int_{\sigma}^{1} \frac{R T}{p} d \sigma$ to accommodate a complicated time integration method that splits the dynamic integration into two energy conserving 
subsystems. The method uses an implicit scheme to solve for $w, p$, and $T$, the Adams-Bashforth scheme for the advection terms, the backward scheme for the pressure gradient force term, and the trapezoidal scheme for the Coriolis term. The WRF-ARW core also adds $\mathrm{w}$ and total pressure $\mathrm{p}$ as two additional prognostic variables, while it adds the third momentum equation and $w g=\frac{d \Phi}{d t}$ together with the gas law to close the system. It integrates the governing equations by a time-split scheme similar to our method described in the previous section except: (i) it uses a third-order RungeKutta scheme in the large time step integration; (ii) it considers moisture in the density calculation; and (iii) it includes the prediction of mass change in the small time step integration for sound waves.

As suggested by Laprise (1992), nonhydrostatic equations formulated with the mass coordinates can be solved by either adding a prognostic equation for pressure or a prognostic equation for the geopotential. Two dynamic cores of the WRF choose to add the prognostic equation for the geopotential, while we choose to add the prognostic equation for pressure. The choice of adding the prognostic equation for pressure makes the terms involved with sound waves easy to identify for formulating the time-split scheme. On the other hand, special care must be made to ensure consistency between pressure and mass prediction. We solve this problem by separating the pressure prediction into mass conservation and $p^{\prime}$ prediction. All three methods discussed here include some kind of linearization in their numerical schemes. We linearize the prognostic equation for $p^{\prime}$, the WRF-ARW core linearizes the gas law when it computes pressure perturbation in the small time step integration, and the WRF-NMM core linearizes the coefficient of a secondorder differential equation solved iteratively to close the implicit scheme for $w$ and $p$. The WRF-NMM core and our method use efficient second-order time schemes in the time integration. Only the WRF-ARW core uses a more expensive higher order time scheme in the time integration. The third-order Runge-Kutta scheme is very accurate in calculating the advection to preserve the shape of the material transported, which is important for cloud and aerosol modeling. However, for NWP applications, as our model is designed for, a second order time scheme is sufficient since major numerical errors come from spatial differencing rather than the time scheme and a relatively small time step is usually required by the CFL linear stability condition.

\section{IDEALIZED CASE TESTS}

We have tested this method of extending a hydrostatic model to a nonhydrostatic model by 2-dimensional (2D) mountain waves and density flows associated with a cold bubble. In these idealized tests, the nonhydrostatic model is modified to be two dimensional with 481 grid points in the horizontal and 87 levels (for mountain wave simulation) or 77 levels (for cold bubble simulation) in the vertical. The model is integrated with dry dynamics only. To control boundary reflection at the model top, we treat the top 15 model layers as sponge layers where second-order vertical diffusion is applied to $u$ and $T$, Rayleigh type damping is applied to $\mathrm{w}$ and $p^{\prime}$, and second-order horizontal diffusion is applied to vertical difference $\delta u$ and $\delta T$ and $p^{\prime}$. Radiative type boundary conditions (Orlanski 1976) are used at lateral boundary points where no gradient is assumed at the inflow boundary and extrapolation is made at the outflow boundary. We treat 25 grid points next to each lateral boundary as a lateral boundary zone where second-order horizontal diffusion is applied to all prognostic variables to control boundary noise. In addition to these boundary treatments, weak diffusion of the fourth-order in the horizontal and secondorder in the vertical are applied at interior grid points.

In mountain wave tests, we first repeat the 2D mountain wave simulations conducted by Dudhia (1993) for testing the MM5. In these numerical simulations, a bell-shaped mountain $h_{s}(x)=h_{0} /\left[1+(x / a)^{2}\right]$ is used. In this expression, $a$ represents the half width and $h_{0}$ represents the maximum height of the mountain. Five different half-width mountains, $100 \mathrm{~m}, 1,10,100$, and $1000 \mathrm{~km}$ together with $h_{0}$ $=400 \mathrm{~m}$ are selected to test the accuracy of the model in simulating five different characters of linear mountain waves discussed in Queney (1948) and Dudhia (1993). As in the MM5 simulations, the grid resolution $\Delta x$ is $1 / 5$ of the half width and the time step is proportional to the grid resolution with $\Delta t=1 \mathrm{~s}$ for $\Delta x=200 \mathrm{~m}$. Coriolis force is included in all five simulations with Coriolis parameter $f=1.0 \mathrm{e}^{-4}$. The initial conditions are in geostrophic and hydrostatic balance with $U_{0}=10 \mathrm{~m} \mathrm{~s}^{-1}$ zonal wind and zero meridional wind everywhere. Temperature and height are initialized with a constant stratification of $N_{0}=\sqrt{\frac{g \partial \theta}{\theta \partial z}}=0.01 \mathrm{~s}^{-1}, 300 \mathrm{~K}$ surface temperature, and $1000 \mathrm{hPa}$ pressure at ground $(z=0)$. These conditions give $N_{0} h_{0} / U_{0}=0.4$, which corresponds to linear mountain waves (Laprise and Peltier 1989). The only difference in our simulation setup is that we use 481 grid points in the horizontal direction and 87 levels in the vertical. The dispersive nature of the nonhydrostatic mountain waves causes large amplitude waves to propagate into model boundaries. As very crude boundary condition treatments applied at the top and lateral boundaries, we need more grid points in the model domain to ensure the boundary noise will be kept far away from the region where we are looking for steady solutions. The vertical resolution is $13 \mathrm{hPa}$ in the bottom 60 levels and is gradually reduced to $7 \mathrm{hPa}$ in top 16 levels. With this selection the sponge layer is above $105 \mathrm{hPa}$ (approximately $12.2 \mathrm{~km}$.)

For convenience in comparison, we plot our simulation results at 2160 time steps in the same way as that presented 
in the MM5 simulations, i.e., 50 grid points in each side of the mountain peak and up to $10 \mathrm{~km}$ from the ground with the $400 \mathrm{~m}$ mountain at the surface. Figure 1 shows the vertical velocity of the five mountain wave simulations from our nonhydrostatic model. The simulations capture the characteristics of the linear mountain waves from theoretical and numerical studies (Queney 1948; Dudhia 1993); that is, that the wave is evanescent for $a=100 \mathrm{~m}$, downstream propagation for $a=1 \mathrm{~km}$, upright for $a=10 \mathrm{~km}$, influenced by the inertial force for $a=100 \mathrm{~km}$, and non-stationary for $a=$ $1000 \mathrm{~km}$. The flow pattern, wave length, and wave amplitude all agree well with the MM5 simulations. To examine the nonhydrostatic effects on these steady mountain waves, we have conducted the same experiments but without nonhydrostatic contributions (Fig. 2). The results clearly show that nonhydrostatic effects are significant mainly for cases with $N_{0} a / U_{0} \leq 1$, as predicted by early theoretical studies (Laprise and Peltier 1989). To check the impact of the free-slip lower boundary condition on the nonhydrostatic mountain waves, we conduct another $1 \mathrm{~km}$ half-width mountain simulation with viscosity. The viscosity is modeled by the surface stress $\bar{\tau}_{s}=C_{d} \vec{V}|\vec{V}|$, with $C_{d}=0.01$, and free atmosphere stress $\bar{\tau}=k \frac{\partial \bar{V}}{\partial \sigma}$, with $k=0.001$, at the bottom $1 / 4$ sigma levels. The viscosity does not change the main characteristics of the mountain wave, except to significantly reduce the wave amplitude (Fig. 3) and make the solution converge very slowly to a quasi-steady state (not shown). The result is consistent with the theoretical studies in that characteristics of linear mountain waves are determined by $U_{0}, N_{0}, a$, and $f$, but not viscosity.

In the second mountain wave test, we compare the surface pressure perturbation of simulated mountain waves with that computed by a linear theory (Queney 1948; Hsu and Sun 2001). Following Hsu and Sun (2001), we use a $10 \mathrm{~m}$ high mountain to integrate our 2D nonhydrostatic model to a steady state and multiply the pressure perturbation by 100 to compare it with the analytic linear solution for a $1000-\mathrm{m}$ high mountain. In this integration, the Coriolis force is not included and the same model setup for 1-km half width without viscosity is used. The integration is made for 12960 time steps to ensure a steady state solution is reached. Since pressure is a prognostic variable in our model, we can easily calculate surface pressure perturbation as the sum of the nonhydrostatic and surface hydrostatic pressure perturbations, i.e., $\delta p_{s}=\left(\pi_{s}-\pi_{s 0}+p^{\prime}\right)$ where $\pi_{s 0}$ is the initial hydrostatic pressure at the surface. The simulated surface pressure perturbation matches well with the analytic solution computed by Hsu and Sun (2001) and the vertical velocity of the linear wave simulation is very similar to the $400-\mathrm{m}$ height solution in Fig. 1b, but the amplitude is about 40 times less (Fig. 4).

To simulate density flows associated with a cold bubble, we set up a numerical experiment following Straka et al. (1993) and Janjic et al. (2001). The initial conditions are specified as an atmosphere at rest with $1000 \mathrm{hPa}$ pressure at the ground and hydrostatic balance above. The initial temperature is specified with a neutral mean stratification of $300 \mathrm{~K}$ potential temperature everywhere and temperature perturbations of a cold bubble, $\Delta T(x, z)=-15 \cos ^{2}(\pi L / 2)$, added at the domain central area of $L<1$, where $L=$ $\sqrt{\left[\left(x-x_{c}\right) / x_{i}\right]^{2}+\left[\left(z-z_{c}\right) / z_{i}\right]^{2}}, x_{c}=0 \mathrm{~m}, z_{c}=3000 \mathrm{~m}, x_{i}=$ $4000 \mathrm{~m}$, and $z_{i}=2000 \mathrm{~m}$. The 77 vertical levels for this test are selected to have 15 sponge layers above $\sigma=0.442$ (approximately $6.5 \mathrm{~km}$ ) with a vertical resolution varying from 5 to $18 \mathrm{hPa}$. We use $100-\mathrm{m}$ horizontal resolution and $0.3 \mathrm{~s}$ time steps in this nonhydrostatic simulation and apply second-order diffusion in both horizontal and vertical directions using the diffusion coefficient $\mathrm{K}=75 \mathrm{~m}^{2} \mathrm{~s}^{-1}$ as in Straka et al. (1993) and Janjic et al. (2001). The cold potential temperature perturbations from our $2 \mathrm{D}$ nonhydrostatic simulations at initial time, 300, 600, and $900 \mathrm{~s}$ are displayed in Fig. 5. The area shown extends from the model domain center to $19.2 \mathrm{~km}$ to the right, and from the ground to $4.8 \mathrm{~km}$. The minor computational noise on zero contours is due to numerical errors associated with the non-positive definite schemes used in our model for NWP applications. The horizontal and vertical wind components after $900 \mathrm{~s}$ are displayed in Fig. 6 with the same setting. The nonhydrostatic simulation agrees well with the results from the two previous studies that the density flow exhibits one rotor after $600 \mathrm{~s}$ and three rotors after $900 \mathrm{~s}$ generated by the KelvinHelmholtz instability. We also check the nonhydrostatic effects on this falling cold bubble by conducting a hydrostatic simulation without the nonhydrostatic components. In this hydrostatic simulation, we have to use a shorter time step of $0.1 \mathrm{~s}$ for the same grid resolution due to very large vertical motions generated at initial periods by assuming the hydrostatic balance. Figures 7 and 8 show the hydrostatic simulations that correspond to Figs. 5 and 6, respectively. No rotors are generated in the model simulation without the nonhydrostatic components. The Richardson number of the hydrostatic simulation after $300 \mathrm{~s}$ is about 0.7 , which is too large for the Kelvin-Helmholtz instability. Hydrostatic approximation severely distorts the cold bubble simulation at the initial period of falling when the downward vertical velocity is accelerated by negative buoyancy. In this early period, the hydrostatic simulation gives too fast falling and too noisy density flows (Fig. 9), as is expected when the hydrostatic assumption is violated.

\section{SUMMARY}

By interpreting the hydrostatic part of pressure as air mass weight above an area, the primitive equations in the sigma-p coordinates can be directly used to form the major 

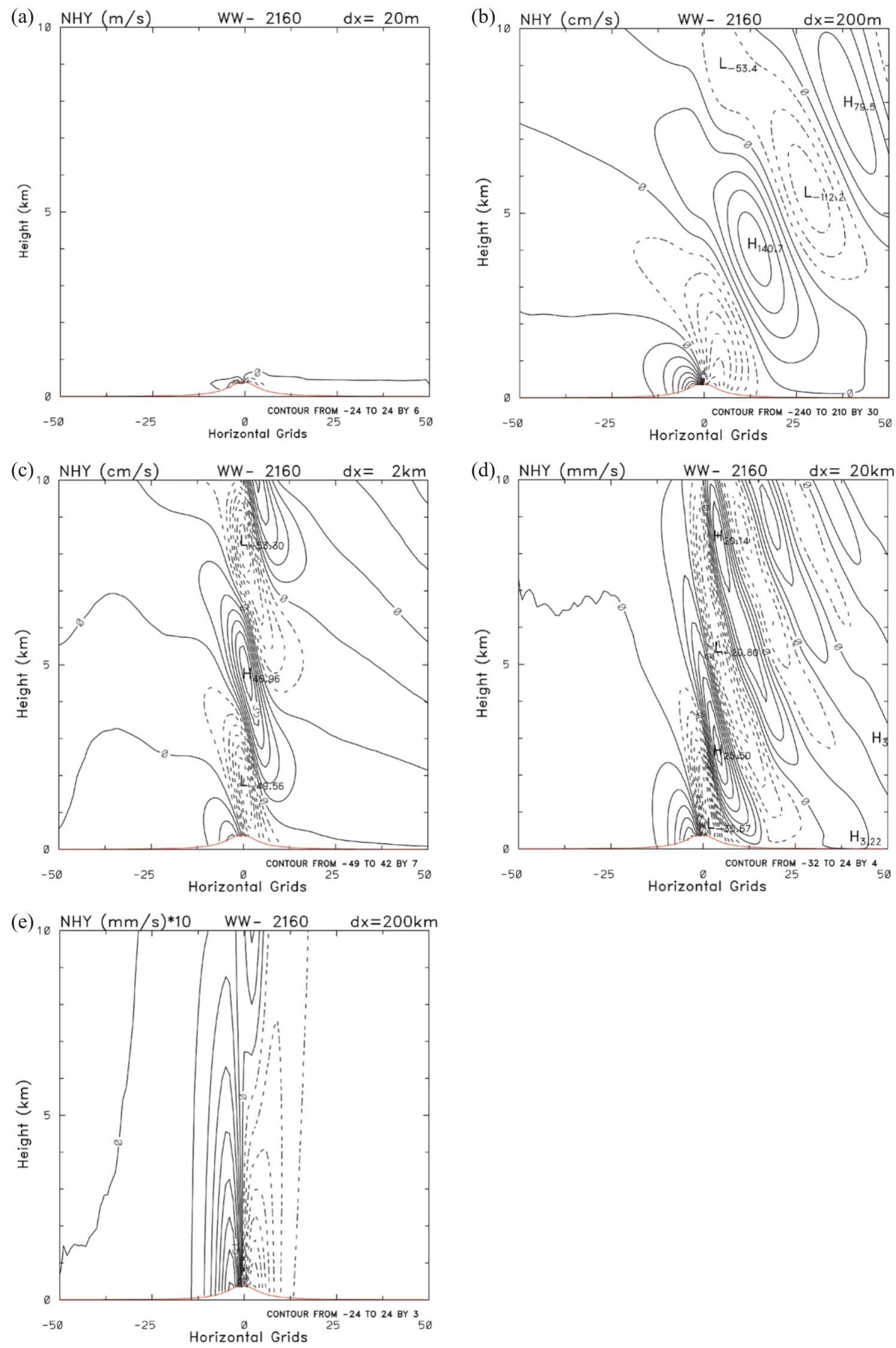

Fig. 1. Vertical velocity of nonhydrostatic and hydrostatic mountain wave simulations after 2160 time steps for 400-m mountains of half-width (a) $100 \mathrm{~m}$, (b) 1, (c) 10, (d) 100, and (e) $1000 \mathrm{~km}$. The contour intervals are $6 \mathrm{~m} \mathrm{~s}^{-1}, 30 \mathrm{~cm} \mathrm{~s}^{-1}, 7 \mathrm{~cm} \mathrm{~s}^{-1}, 4 \mathrm{~mm} \mathrm{~s}^{-1}$, and $0.3 \mathrm{~mm} \mathrm{~s}$ with dash contours for negatives. 

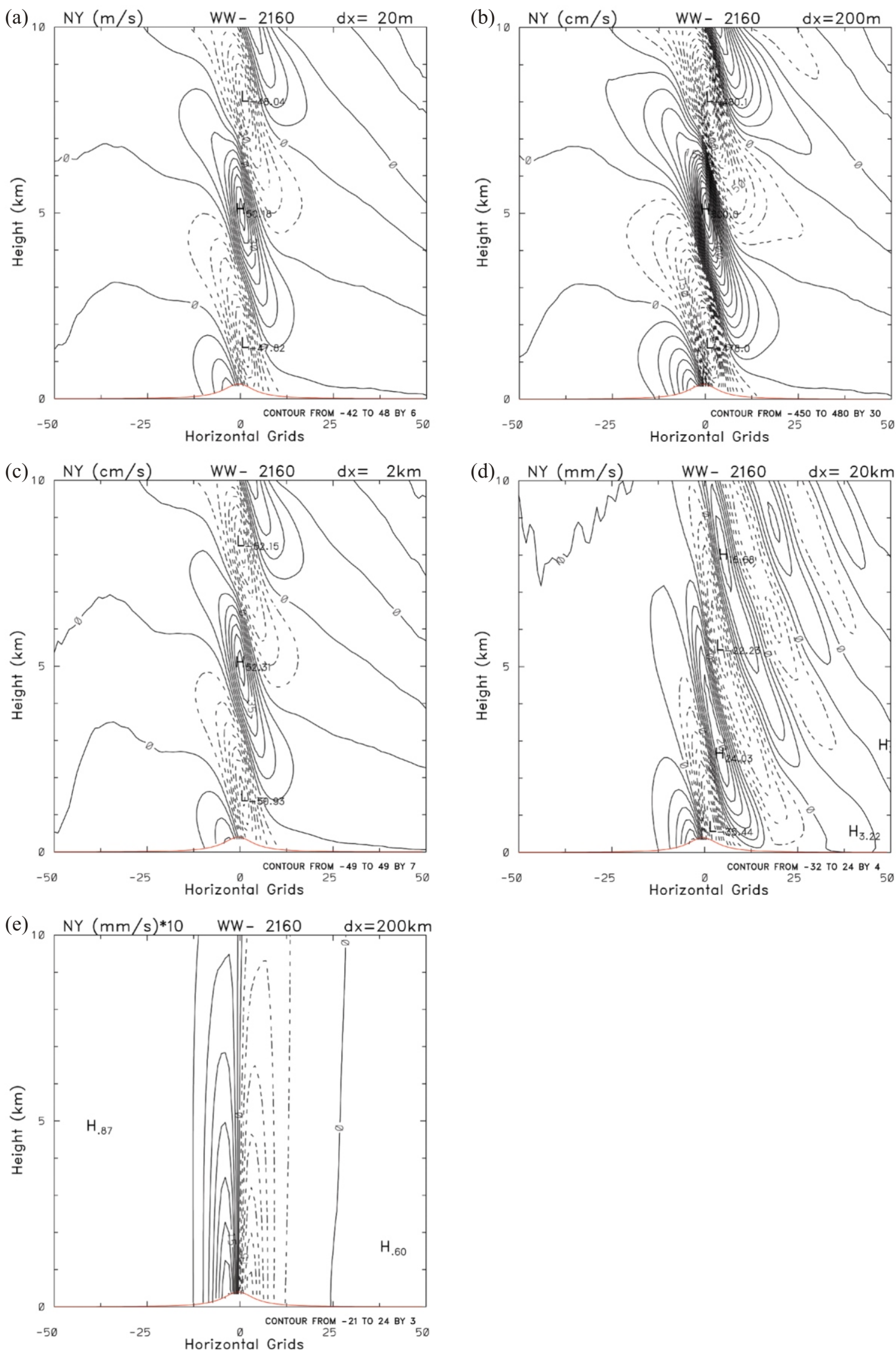

Fig. 2. Same as Fig. 1, except for hydrostatic simulations without the nonhydrostatic contributions. 

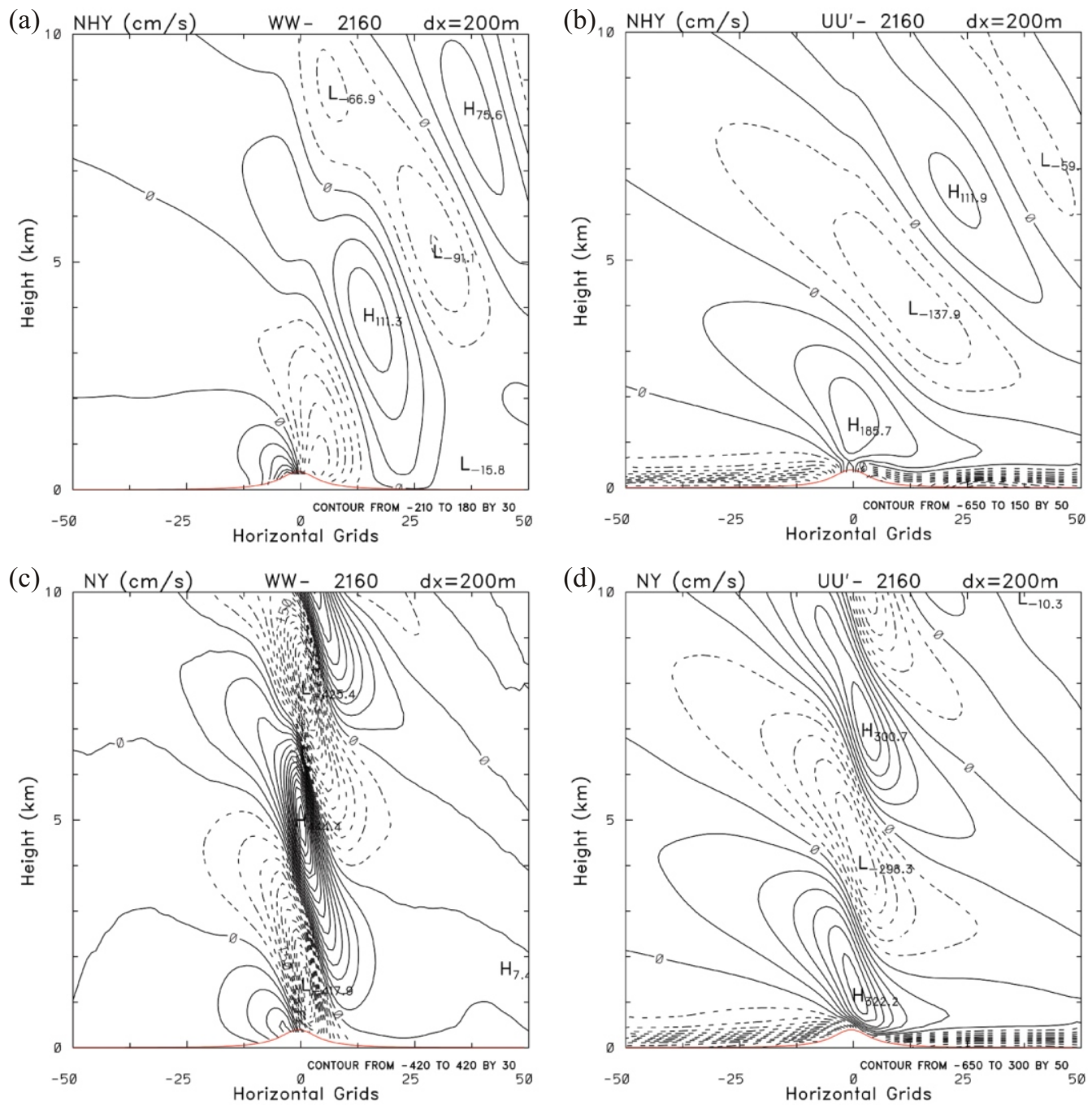

Fig. 3. Mountain wave simulations with viscosity for 1-km half-width hill at 2160 time steps: (a) vertical velocity from nonhydrostatic, (b) zonal wind perturbation from nonhydrostatic, (c) vertical velocity from hydrostatic, and (d) zonal wind perturbation from hydrostatic runs. The zonal wind perturbation is the wind deviation from initial $10 \mathrm{~m} \mathrm{~s}^{-1}$ mean wind. Contour intervals are $30 \mathrm{~cm} \mathrm{~s}^{-1}$ for vertical velocity and $50 \mathrm{~cm} \mathrm{~s}$ for zonal wind perturbation.
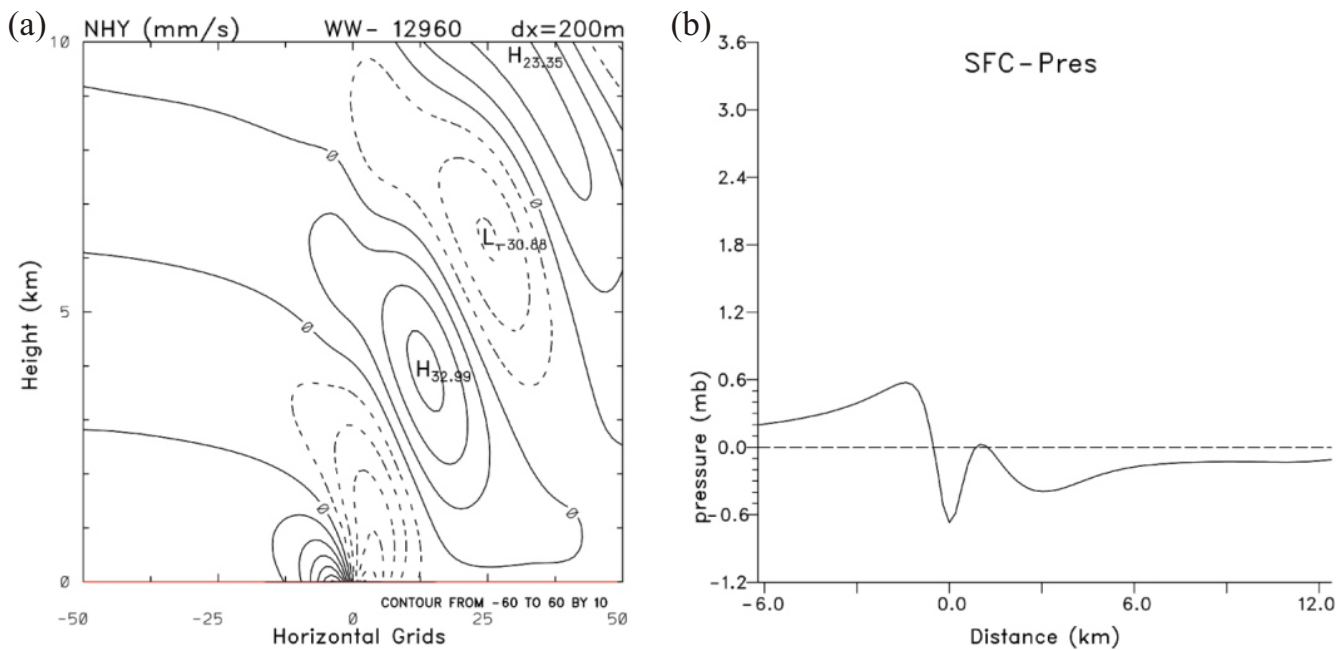

Fig. 4. Linear mountain wave simulation for 1-km half-width hill at 12960 time steps: (a) vertical velocity and (b) surface pressure perturbation around the mountain peak. Contour interval is $10 \mathrm{~mm} \mathrm{~s}^{-1}$ for the vertical velocity. 

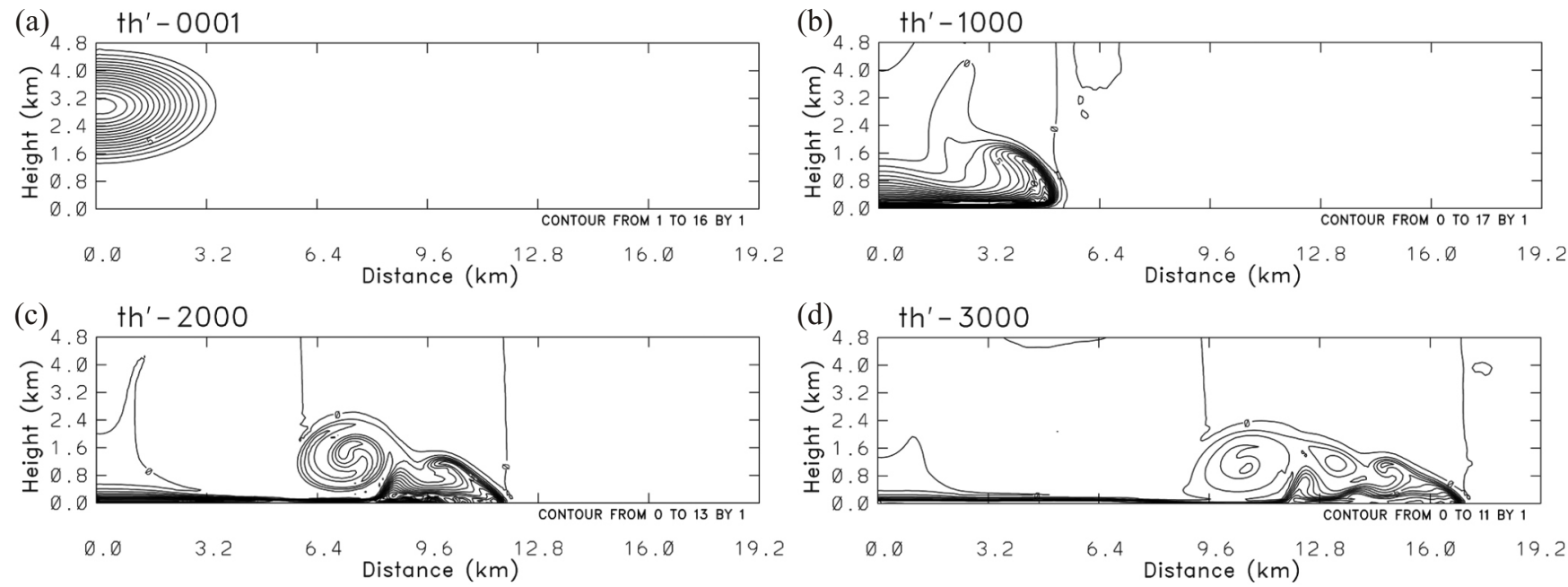

Fig. 5. Cold potential temperature perturbation of the cold bubble simulation with all nonhydrostatic components included at: (a) 0 , (b) 300 , (c) 600, and (d) $900 \mathrm{~s}$. The contour interval is $1 \mathrm{~K}$.
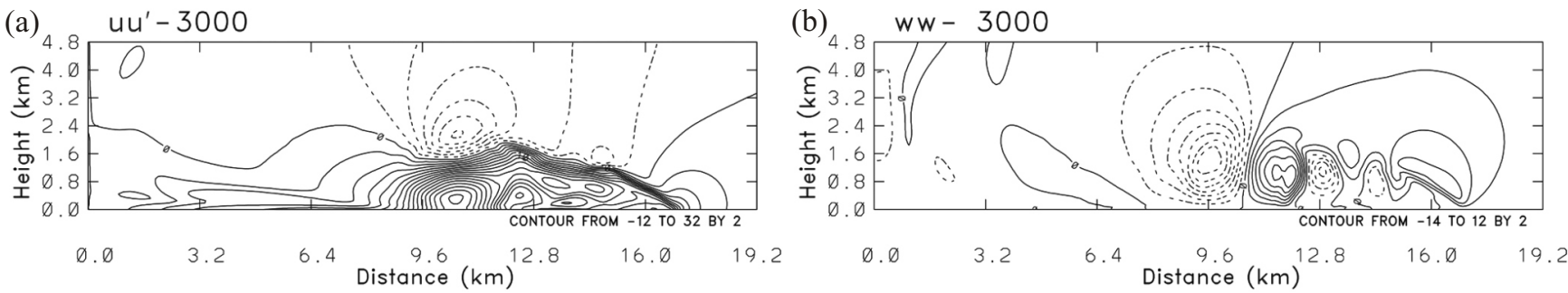

Fig. 6. (a) Zonal wind and (b) vertical wind of the cold bubble simulation with all nonhydrostatic components included at $900 \mathrm{~s}$. The contour interval is $2 \mathrm{~m} \mathrm{~s}^{-1}$ with dashed contours for negative values.
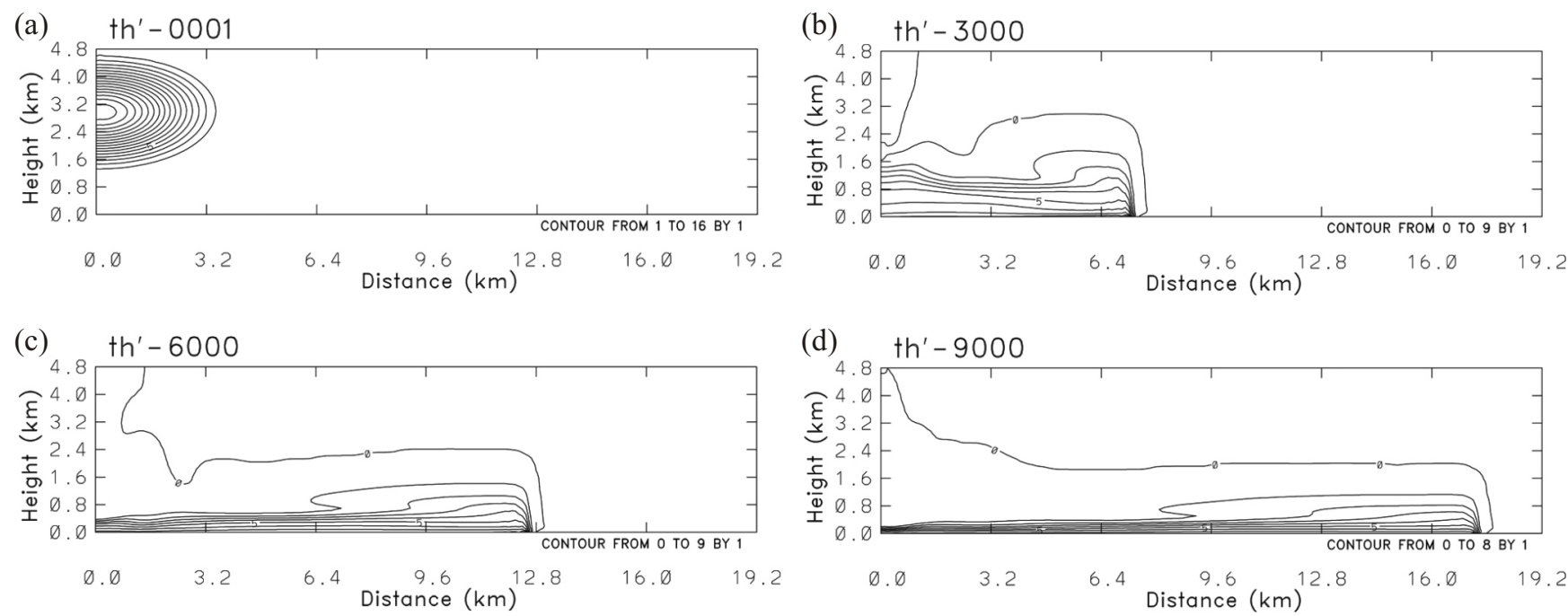

Fig. 7. Same as Fig. 5, except for the hydrostatic simulation without nonhydrostatic components. 


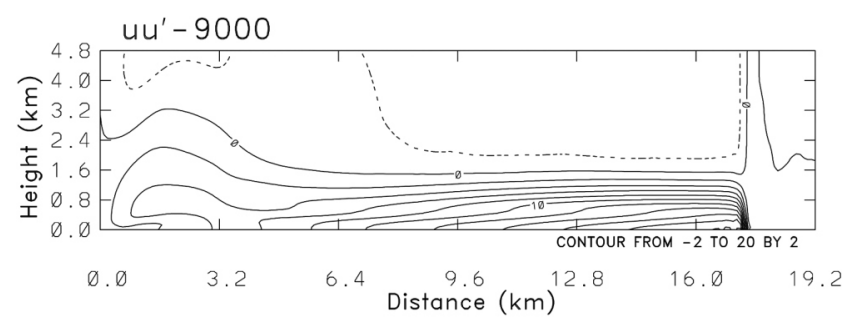

Fig. 8. Same as Fig. 6a, except for the zonal wind of the hydrostatic simulation without nonhydrostatic components.
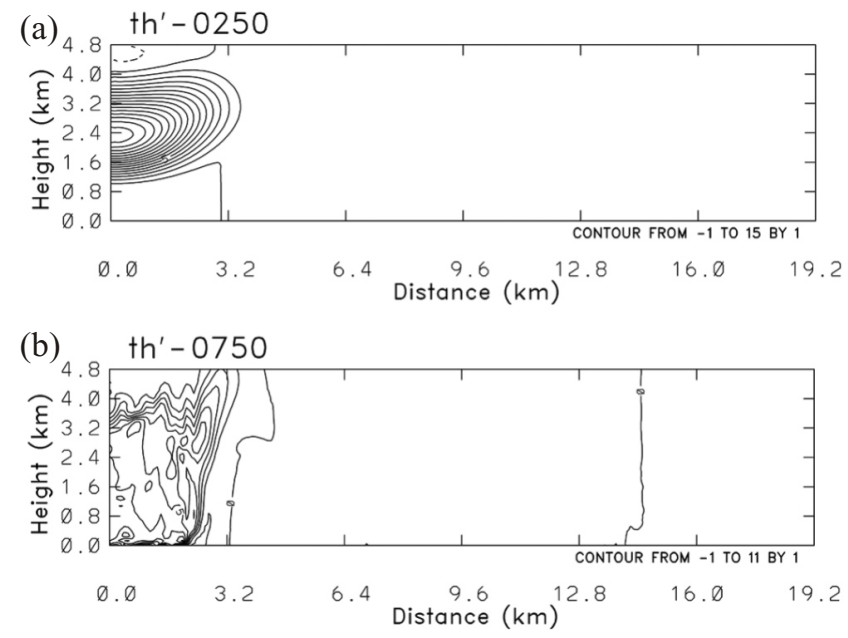

Fig. 9. Cold potential temperature perturbation of the cold bubble simulation at $75 \mathrm{~s}$ for (a) nonhydrostatic and (b) hydrostatic simulations. The contour interval is $1 \mathrm{~K}$.

part of the governing equations in mass coordinates for a fully compressible atmosphere without the hydrostatic approximation. After adding extra terms to the primitive equations and two more equations for $\mathrm{w}$ and $p^{\prime}$ predictions, a hydrostatic model can be easily upgraded to a nonhydrostatic model. The only simplification made in this numerical method is the linearization of the pressure tendency equation, which may distort sound wave simulations but has negligible impact on weather forecasts. With this approach, almost all codes of a hydrostatic model can be used in upgrading the model to a nonhydrostatic model. In our efforts to develop a nonhydrostatic model at the Central Weather Bureau (CWB) of Taiwan, in additional to modifying the model driver and output routines to account for extra variables and routine calls, we only need to add two more routines to compute the right hand side terms of $R H_{()}$for Eqs. (22) to (26) and perform smaller time step integration for Eqs. (22) to (26). The idealized case tests show that the proposed method can realistically simulate nonhydrostatic effects on different atmospheric circulations, which are revealed in theoretical solutions and simulations from other nonhydrostatic models. The nonhydrostatic mesoscale mo- del developed by this method at the CWB, Nonhydrostatic Forecast System (NFS), has been operational since December 2003 (Leou 2004). The NFS is currently running twice (or 4 times when a typhoon is approaching Taiwan) a day with triple nests of $45 / 15 / 5 \mathrm{~km}$ resolutions for mesoscale and typhoon forecasts. This method can also be used in upgrading hydrostatic global models to nonhydrostatic global models.

Acknowledgements The major part of this work was done between 1999 and 2003 during many visits by the first author to the CWB. The authors appreciate the support by the former director general, Mr. Shinn-Liang Shieh.

\section{REFERENCES}

Dudhia, J., 1993: A nonhydrostatic version of the Penn StateNCAR mesoscale model: Validation tests and simulation of an Atlantic cyclone and cold front. Mon. Wea. Rev., 121, 1493-1513, doi: 10.1175/1520-0493(1993)121<1493: ANVOTP $>2.0 . C O ; 2$. [Link $]$

Hodur, R. M., 1997: The Naval Research Laboratory's Coupled Ocean/Atmosphere Mesoscale Prediction System (COAMPS). Mon. Wea. Rev., 125, 1414-1430, doi: 10.1175/ 1520-0493(1997)125<1414:TNRLSC > 2.0.CO;2. [Link]

Hsu, W. R. and W. Y. Sun, 2001: A time-split, forward-backward numerical model for solving a nonhydrostatic and compressible system of equations. Tellus, 53A, 279-299.

Janjic, Z. I., J. P. Gerrity Jr., and S. Nickovic, 2001: An alternative approach to nonhydrostatic modeling. Mon. Wea. Rev., 129, 1164-1178, doi: 10.1175/1520-0493(2001)129 $<$ 1164:AAATNM>2.0.CO;2. [Link]

Kasahara, A., 1974: Various vertical coordinate systems used for numerical weather prediction. Mon. Wea. Rev., 102, 509522, doi: 10.1175/1520-0493(1974)102<0509:VVCSUF $>$ 2.0.CO;2. [Link]

Klemp, J. B., W. C. Skamarock, and J. Dudhia, 2007: Conservative split-explicit time integration methods for the compressible nonhydrostatic equations. Mon. Wea. Rev., 135, 2897-2913, doi: 10.1175/MWR3440.1. [Link]

Laprise, R., 1992: The Euler equation of motion with hydrostatic pressure as an independent variable. Mon. Wea. Rev., 120, 197-207, doi: 10.1175/1520-0493(1992)120<0197: TEEOMW $>2.0 . \mathrm{CO} ; 2$. [Link]

Laprise, R and W. R. Peltier, 1989: On the structural characteristics of steady finite-amplitude mountain waves over bell-shaped topography. J. Atmos. Sci., 46, 586-595, doi: 10.1175/1520-0469(1989)046<0586:OTSCOS>2.0.CO;2. [Link]

Leou, T. M., 2004: Operational status and future plans of Nonhydrostatic Forecast System at CWB: Precipitation treatment. Proceedings of Conference on Weather Analysis and Forecasting, May 2004, Taipei, Taiwan, 229234.

Madala, R. V., 1981: Efficient time integration schemes for at- 
mosphere and ocean models. In: Book, D. L. (Ed.), Finite-Difference Techniques for Vectorized Fluid Dynamic Calculations, Spring-Verlag, 56-70.

Orlanski, I., 1976: A simple boundary condition for unbounded hyperbolic flows. J. Comput. Phys., 21, 251-269, doi: 10.1016/0021-9991(76)90023-1. [Link]

Queney, P., 1948: The problem of airflow over mountains: A summary of theoretical studies. Bull. Amer. Meteor. Soc., 29, 16-26.

Straka, J. M., R. B. Williamson, L. J. Wicker, J. R. Anderson, and K. K. Droegemeier, 1993: Numerical solutions of a non-linear density current: A benchmark solution and comparisons. Int. J. Numer. Methods Fluids, 17, 1-22, doi: 10.1002/fld.1650170103. [Link]

\section{APPENDIX}

The pressure tendency equation expressed as:

$$
\frac{d \ln p}{d t}+\frac{C_{p}}{C_{v}} D_{3}=\frac{Q_{T}}{C_{v} T}
$$

can be derived by taking the ' $\delta$ ln' operator on the equation of state $p=\rho R T$ together with the continuity equation,

$\frac{d \ln \rho}{d t}+D_{3}=0$

and the first law of thermodynamics,

$\frac{d \ln T}{d t}+\frac{R}{C_{v}} D_{3}=\frac{Q_{T}}{C_{v} T}$

In a general vertical coordinate $\sigma$, the 3 -dimensional divergence $D_{3}$ can be written as:

$D_{3}=\nabla \cdot \vec{V}-\frac{\partial \sigma}{\partial z} \frac{\partial \vec{V}}{\partial \sigma} \cdot \nabla z+\frac{\partial w}{\partial \sigma} \frac{\partial \sigma}{\partial z}$

$=\nabla \cdot \vec{V}-\frac{\partial \sigma}{\partial z} \frac{\partial \vec{V}}{\partial \sigma} \cdot \nabla z+\frac{\partial \sigma}{\partial z} \frac{\partial}{\partial \sigma} \frac{d z}{d t}$

$=\nabla \cdot \vec{V}-\frac{\partial \sigma}{\partial z} \frac{\partial \vec{V}}{\partial \sigma} \cdot \nabla z+\frac{\partial \sigma}{\partial z}\left(\frac{d}{d t} \frac{\partial z}{\partial \sigma}+\frac{\partial \vec{V}}{\partial \sigma} \cdot \nabla z+\frac{\partial \dot{\sigma}}{\partial \sigma} \frac{\partial z}{\partial \sigma}\right)$

$=\nabla \cdot \vec{V}-\frac{\partial \sigma}{\partial z} \frac{\partial \vec{V}}{\partial \sigma} \cdot \nabla z+\frac{d}{d t}\left(\ln \frac{\partial z}{\partial \sigma}\right)+\frac{\partial \sigma}{\partial z} \frac{\partial \vec{V}}{\partial \sigma} \cdot \nabla z+\frac{\partial \dot{\sigma}}{\partial \sigma}$

$=\nabla \cdot \vec{V}+\frac{\partial \dot{\sigma}}{\partial \sigma}+\frac{d}{d t}\left(\ln \frac{\partial z}{\partial \sigma}\right)$
In both sigma-p and mass coordinates, we find $\frac{\partial z}{\partial \sigma}=-\frac{\rho g}{\pi_{s}}$ since $\delta p=\pi_{s} \delta \sigma=-\rho g \delta z$ in the sigma-p coordinate and $\delta m$ $=\pi_{s} \delta \sigma=-\rho g \delta z$ in the mass coordinate. Therefore, in both coordinate systems, $D_{3}$ can be expressed as:

$D_{3}=\nabla \cdot \vec{V}+\frac{\partial \dot{\sigma}}{\partial \sigma}-\frac{d \ln \rho}{d t}+\frac{d \ln \pi_{s}}{d t}$

with which the continuity Eq. (A2) can be written as:

$\frac{d \ln \pi_{s}}{d t}+\nabla \cdot \vec{V}+\frac{\partial \dot{\sigma}}{\partial \sigma}=0$

Under hydrostatic approximation the pressure is express as $p=\pi_{s} \sigma$. We can apply ' $\delta \ln$ ' to $p=\rho R T$ and get $\delta \ln \pi_{s}+$ $\delta \ln \sigma=\delta \ln \rho+\delta \ln T$. Therefore, with the hydrostatic approximation, $D_{3}$ in Eq. (A5) can be written as:

$D_{3}=\nabla \cdot \vec{V}+\frac{\partial \dot{\sigma}}{\partial \sigma}-\frac{\dot{\sigma}}{\sigma}+\frac{d \ln T}{d t}$

Substituting Eq. (A7) into Eq. (A1), the pressure tendency equation with the hydrostatic approximation can then be expressed as:

$$
\begin{aligned}
& \frac{d\left(\ln \pi_{s}+\ln \sigma\right)}{d t}+\left(\nabla \cdot \vec{V}+\frac{\partial \dot{\sigma}}{\partial \sigma}-\frac{\dot{\sigma}}{\sigma}+\frac{d \ln T}{d t}\right) \\
& +\frac{R}{C_{v}} D_{3}=\frac{Q_{T}}{C_{v} T},
\end{aligned}
$$

or

$$
\frac{d \ln \pi_{s}}{d t}+\nabla \cdot \vec{V}+\frac{\partial \dot{\sigma}}{\partial \sigma}+\frac{d \ln T}{d t}+\frac{R}{C_{v}} D_{3}=\frac{Q_{T}}{C_{v} T}
$$

which is a trivial equation since it is simply the sum of the continuity Eq. (A6) and thermodynamic Eq. (A4). It is consistent with the fact that under hydrostatic approximation the pressure can be diagnosed by $p=\pi_{\mathrm{s}} \sigma$ without a prognostic equation. 